\title{
Self-interacting neutrino portal dark matter
}

\author{
J. M. Lamprea* \\ Fac. de Cs. de la Electrónica, Benemérita Universidad Autónoma de Puebla, \\ A.P. 542, Puebla, Pue. 72000, México \\ E. Peinado ${ }^{\dagger}$ \\ Instituto de Física, Universidad Nacional Autónoma de México, \\ A.P. 20-364, Ciudad de México 01000, México \\ S. Smolenski $i^{\ddagger}$ and J. Wudka $\odot^{\S}$ \\ Department of Physics and Astronomy, UC Riverside, Riverside, California 92521-0413, USA
}

(Received 14 October 2019; revised 20 October 2020; accepted 16 November 2020; published 14 January 2021)

\begin{abstract}
We present a realistic, simple, and natural model of self-interacting dark matter based on the neutrinoportal paradigm. The self-interactions at small velocities are generated by the exchange of dark photons and produce the observed core-like DM distribution in galactic centers. This effect could be spoiled by the formation of DM bound states (also due to dark-photon effects), which we avoid by requiring the DM candidates to be light, with masses below $O(10 \mathrm{GeV})$. The mixing of the dark photon with the $Z$ and ordinary photon is strongly suppressed by introducing a softly broken discrete symmetry similar to charge conjugation, which also ensures that the dark photon lifetime is short enough to avoid restrictions derived form big-bang nucleosynthesis and large-scale structure formation. Other constraints are accommodated without the need of fine tuning, in particular nucleon scattering occurs only at one loop, so direct detection cross sections are naturally suppressed. Neutrino masses are generated through the inverse seesaw.
\end{abstract}

DOI: 10.1103/PhysRevD.103.015017

\section{INTRODUCTION}

The nature of dark matter (DM) remains one of the most perplexing problems in modern particle and astroparticle physics. Current evidence for the existence of massive particles that interact weakly with the Standard Model (SM) is entirely gravitational [1-5], and every attempt at direct [6-12], indirect [13-23] or collider [24-26] detection has only led to increasingly stronger constraints on models. Besides, estimations of the DM distribution in some darkmatter-dominated galaxies indicate that the DM density at the core does not exhibit a spike and is less dense as would be expected if it behaved as an ideal gas. This cusp-core problem [27-32] belongs to the so-called small scale problem in the $\Lambda \mathrm{CDM}$ cosmology [33] accounting for the mass deficit in small-scale halos. Such small-scale

\footnotetext{
*jmlamprea@gmail.com epeinado@fisica.unam.mx

*ssmol001@ucr.edu

§ose.wudka@ucr.edu
}

Published by the American Physical Society under the terms of the Creative Commons Attribution 4.0 International license. Further distribution of this work must maintain attribution to the author(s) and the published article's title, journal citation, and DOI. Funded by SCOAP. problems arise from the disparities between $\Lambda \mathrm{CDM}$ $\mathrm{N}$-body simulation and the observed substructure in the Universe, which also includes the "missing satellite" [34,35] and "too-big-to-fail" [36-39] problems. Such discrepancies may be due to systematic errors in the DM distribution profiles extracted from observation (see, e.g., [40-44]) or the lack of understanding of dissipative baryonic effects, such as gas cooling, star formation and supernovae feedback in galactic evolution (see, e.g., [45]). Alternatively, these problems could be alleviated by new DM dynamics as warm DM or self-interacting dark matter (SIDM). Strong and velocity-dependent self-interactions $[46,47]$ within the dark sector, realized by a light mediator, can address the small-scale problem and remain consistent with constraints derived from galaxy cluster mergers.

In this paper we will discuss a simple SIDM model that meets all available constraints without fine tuning of parameters. The model is an extension of one discussed earlier $[48,49]$, based on the neutrino-portal paradigm [48-65] where the dark sector couples to the SM via (Dirac) fermion mediators that mix with the SM neutrinos. The dark sector contains two quasidegenerate fermions, which constitute the relic density, and a scalar, more massive than the fermions. Interactions within the dark sector are mediated by a dark photon, whose mixing with the ordinary photon is (again, naturally) strongly suppressed, occurring 
at three loops; the main decay mode of the dark photon is into neutrinos, and appears at one loop, so the dark photon is relatively long-lived. In contrast with the earlier model, we find that the main DM annihilation channel is into 2 dark photons, leading eventually to 4 neutrinos. If the inclusion of self-interaction in the dark sector is the explanation for the small-scale problem the DM mass is bounded from above, otherwise it starts forming bound states.

The paper is organized as follows: in the next section we describe the model, concentrating on the interactions within the dark sector; detailed discussion of the other aspects can be found in [48]. In Secs. III, IV and V we discuss the electroweak, relic abundance, and direct-detection constraints, respectively. Section VII contains results from numerical simulations, and we present our conclusions in Sec. VIII.

\section{THE MODEL}

As noted above, we will study an extension of the neutrino portal dark matter model discussed in [48], where we add self-interactions to the dark sector, and double the number of fermions (the justification for this is provided below). The dark sector then contains two fermions $\Psi_{ \pm}$with masses $m_{ \pm}$, and one complex scalar $\Phi$ that we assume heavier than the fermions, $m_{\Phi}>m_{ \pm}$. In this case we have a fermionic DM candidate. The dark sector is connected to the Standard Model through a set of three Dirac neutral fermionic mediators $\mathcal{F}$, with interactions of the form $\bar{\Psi} \Phi \mathcal{F}$ and ${ }^{1} \bar{l} \mathcal{F} \tilde{\phi}$.

We generate interactions within the dark sector by assuming the presence of a $U(1)_{\text {dark }}$ gauge symmetry under which $\Psi_{ \pm}$and $\Phi$ are charged; we denote by $V$ the corresponding gauge boson, the dark photon. We will assume the $V$ has a non-zero mass $m_{\mathrm{V}}$ that we introduce using the Stückelberg trick. As we show below, the cross sections generated by $V$ exchange have the velocity dependence [46] required to address the core-cusp problem. The presence of the $V$ also resolves a tension with the relic abundance that occurs when the fermions are light $\left(m_{\Psi} \lesssim 10 \mathrm{GeV}\right)$, while the mass splitting of the fermions allows $V$ to decay, which prevents problems with constraints derived from large-structure formation and bigbang nucleosynthesis.

Models with a dark photon contain a kinetic mixing term of the form $\xi V_{\mu \nu} B^{\mu \nu}$ [66], where $B$ is the Standard Model hypercharge gauge field. The coupling $\xi$ is strongly constrained by data: $\xi \leq 10^{-3}$ [67], which we interpret as an indication that the model should contain a symmetry that forbids this interaction and which is either exact or softly broken. For this reason, we impose a dark $\mathbb{Z}_{2}$ symmetry (that we call dark charge conjugation-DCC) under which all SM

\footnotetext{
${ }^{1} l$ denotes the Standard Model left-handed lepton isodoublet, $\phi$ the Higgs isodoublet and $\tilde{\phi}=i \sigma_{2} \phi^{*}$, with $\sigma_{2}$ the usual Pauli matrix; $l$ and $\mathcal{F}$ carry a family index that we suppress.
}

particles and fermionic mediators $\mathcal{F}$ are even, $V$ is odd, the dark scalar has the expected $\Phi \rightarrow \Phi^{*}$ behavior, and $\left\{\Psi_{+}, \Psi_{-}\right\}$ form a dark-charged doublet exchanged under DCC:

$$
\text { DCC : } \Psi_{+} \leftrightarrow \Psi_{-}, \quad \Phi \leftrightarrow \Phi^{*}, \quad V \leftrightarrow-V .
$$

The DCC symmetry requires that $\Psi_{+}$and $\Psi_{-}$have the same mass and couplings, and it also implies that a sufficiently light $V$ will be stable, ${ }^{2}$ which is phenomenologically troublesome. For this last reason, we will assume that DCC is softly broken by assuming the $\Psi_{ \pm}$masses are split; this is the only way to achieve this soft breaking with the particle content we assume. The $V$ couples to the neutrino sector at one loop, to the $Z$ at two loops and to the photon at 3 loops; these features allow the model to be phenomenologically viable without fine tuning. ${ }^{3}$

The Lagrangian for this model is given by

$$
\begin{aligned}
\mathcal{L}= & \mathcal{L}_{\mathrm{SM}}+\bar{\Psi}_{+}\left(i \not D_{+}-m_{+}\right) \Psi_{+}+\bar{\Psi}_{-}\left(i \not D_{-}-m_{-}\right) \Psi_{-}+\left|D_{+} \Phi\right|^{2} \\
& -\frac{1}{2} m_{\Phi}^{2}|\Phi|^{2}-\frac{1}{4} \lambda|\Phi|^{4}-\frac{1}{4} V_{\mu \nu} V^{\mu \nu}+\frac{1}{2} m_{\mathrm{V}}^{2}\left(V_{\mu}-\frac{1}{m_{\mathrm{V}}} \partial_{\mu} \sigma\right)^{2} \\
& +\overline{\mathcal{F}}\left(i \not \partial-m_{\mathcal{F}}\right) \mathcal{F}+\left[\overline{\mathcal{F}} M_{\mathrm{Maj}} \mathcal{F}^{c}+\text { H.c. }\right] \\
& -\left[\bar{l} Y^{(\nu)} \mathcal{F} \tilde{\phi}+\text { H.c. }\right]-\left[\left(\bar{\Psi}_{+} \Phi+\bar{\Psi}_{-} \Phi^{*}\right)(z \mathcal{F})+\text { H.c. }\right] \\
& -\lambda_{x}|\Phi|^{2}|\phi|^{2},
\end{aligned}
$$

where $\mathcal{L}_{\mathrm{SM}}$ is the SM Lagrangian while $l$ and $\phi$ are, respectively, the SM left-handed lepton and scalar isodoublets; also

$$
D_{ \pm}^{\alpha}=\partial^{\alpha} \pm i g_{\mathrm{V}} V^{\alpha}
$$

is the dark covariant derivative, and

$$
m_{ \pm}=m_{\Psi} \pm \mu,
$$

where $\mu$, the fermion mass splitting, parametrizes the soft breaking of DCC; $\sigma$ is the auxiliary field used in the Stückelberg trick (the unitary gauge corresponds to $\sigma=0$ ), and $M_{\text {Maj }}$ is a small Majorana mass term for the $\mathcal{F}$. Finally, we assume three $\mathcal{F}$ fields, ${ }^{4}$ hence $m_{\mathcal{F}}$ and $Y^{(\nu)}$ are $3 \times 3$ mass and Yukawa coupling matrices, respectively, and $z$ is a $3 \times 1$ vector.

Once the Standard Model $S U(2)$ gauge symmetry is broken, the neutrinos $\nu_{L}$ (contained in $l$ ) will mix with the

\footnotetext{
${ }^{2}$ There are viable models with stable light mediator avoiding the CMB and LSS constraints (e.g., [68,69]); these, however, allow for the annihilation of the vectors into dark scalars that, in turn, annihilate into SM particles. Such channels do not exist in the present model.

${ }^{3}$ Except for the usual naturality problems of nonsupersymmetric scalar sectors and those where the light scalars are pseudoGoldstone bosons.

${ }^{4}$ We have suppressed all family indices.
} 

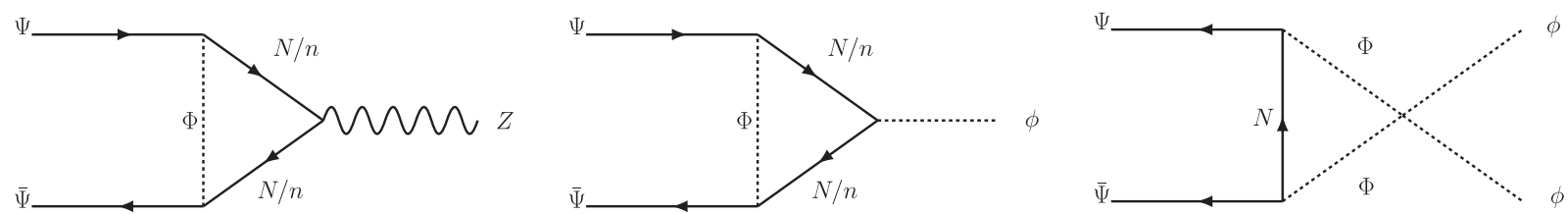

FIG. 1. Loop graphs generating the $\Psi \Psi Z$ and $\Psi \Psi H$ couplings.

$\mathcal{F}$; we will denote the mass eigenstates as $n_{L}$, left-handed with Majorana masses of order $M_{\text {Maj }}\left[Y^{(\nu)} v_{\mathrm{H}} / m_{\mathcal{F}}\right]^{2}$ (where $v_{\mathrm{H}}$ is the SM vacuum expectation value), characteristic of the inverse seesaw mechanism [70-72]; and $N$, with masses of order $m_{\mathcal{F}}$. To reduce the number of parameters, we will assume for simplicity that the $N$ are degenerate, ${ }^{5}$ with mass $m_{\mathrm{N}}$. In this case, ignoring $M_{\mathrm{Maj}}$ (see below), the gauge and mass eigenstates are related by

$$
\begin{aligned}
\mathcal{F} & =\mathcal{C} N_{L}+\mathcal{S} n_{L}+N_{R}, \\
\nu & =V_{\mathrm{PNNS}}^{\dagger}\left(\mathcal{C} n_{L}-\mathcal{S} N_{L}\right),
\end{aligned}
$$

where $V_{\text {PMNS }}$ is the usual PMNS matrix, and $\mathcal{S}$ and $\mathcal{C}$ are real, diagonal $3 \times 3$ mixing matrices that obey

$$
\mathcal{S}^{2}+\mathcal{C}^{2}=\mathbb{1} .
$$

In terms of these quantities,

$$
m_{\mathcal{F}}=m_{\mathrm{N}} \mathcal{C}, \quad Y^{(\nu)}=\sqrt{2} \frac{m_{\mathrm{N}}}{v_{\mathrm{H}}} V_{\mathrm{PMNS}}^{\dagger} \mathcal{S}
$$

The $M_{\text {Maj }}$ term represent a soft and explicit breaking of lepton number, so the smallness of the neutrino masses is (technically) natural; since this mass matrix is arbitrary, it can be adjusted to generate the observed masses and mixing angles in the neutrino sector. The $M_{\mathrm{Maj}}$ term has negligible effects on the observables we discuss in this paper, and for this reason we will ignore it in the following discussion. As a last simplification, we will assume that the $z$ Yukawa couplings are real, as the model observables we consider here only depend on the magnitude of $z_{i}$; in this case the model has 11 parameters: $\left\{m_{\mathrm{N}}, m_{\Psi}, m_{\Phi}, \mu\right\}(4),\left\{\lambda_{x}, z, \mathcal{S}\right\}(7)$.

The various interaction terms involving the $n_{L}$ and $N$ take the form

$$
\begin{aligned}
& Z \text { couplings: }-\frac{g}{2 c_{\mathrm{W}}}\left[\bar{n}_{L} \mathcal{C}^{2} \not n_{L}+\bar{N}_{L} \mathcal{S}^{2} \not Z N_{L}+\left(\bar{n}_{L} \mathcal{C} \mathcal{S} \not Z N_{L}+\text { H.c. }\right)\right] \text {. } \\
& W \text { couplings: }-\frac{g}{\sqrt{2}}\left[\bar{e} W V_{\mathrm{PMNS}}^{\dagger} \mathcal{C} n_{L}-\bar{e} W V_{\mathrm{PMNS}}^{\dagger} \mathcal{S} N_{L}+\text { H.c. }\right] \text {. } \\
& \text { Yukawa couplings: }+\frac{m_{\mathrm{N}}}{v_{\mathrm{H}}} H\left[\bar{N}_{R} \mathcal{S C} n_{L}-\bar{N}_{R} \mathcal{S}^{2} N_{L}+\text { H.c. }\right] \text {. } \\
& \text { DM couplings: }+\left[\bar{\Psi}_{ \pm} \Phi z \mathcal{S} n_{L}+\bar{\Psi}_{ \pm} \Phi z\left(\mathcal{C} P_{L}+P_{R}\right) N+\text { H.c. }\right]+\lambda_{x}|\phi|^{2}|\Phi|^{2} \text {. }
\end{aligned}
$$

As a matter of notation we find it convenient to define

$$
r_{i j}=\left(\frac{m_{i}}{m_{j}}\right)^{2},
$$

so that $r_{\mathrm{NZ}}=\left(m_{\mathrm{N}} / m_{\mathrm{Z}}\right)^{2}$, etc.

\section{A. Loop-induced couplings}

The above model has no tree-level couplings of the DM ( $\Psi)$ to the $Z$ and $H$ bosons. These couplings are generated at one loop by the graphs in Fig. 1. Assuming zero external momenta a straightforward calculation gives [48]

\footnotetext{
${ }^{5}$ This degeneracy is broken by the Majorana mass term in Eq. (2), which we assume small.
}

$$
\begin{aligned}
& \mathcal{L}_{\mathrm{DM}-\mathrm{Z}}=-\frac{g}{2 c_{\mathrm{W}}} \bar{\Psi}_{ \pm} \not\left(\epsilon_{L} P_{L}+\epsilon_{R} P_{R}\right) \Psi_{ \pm} \\
& \mathcal{L}_{\mathrm{DM}-\mathrm{H}}=\epsilon_{H} \bar{\Psi}_{ \pm} \Psi_{ \pm} H
\end{aligned}
$$

where [see Eq. (9)]

$$
\begin{aligned}
\epsilon_{R}= & -\frac{\left(z \mathcal{S}^{2} \mathcal{C}^{2} z^{T}\right)}{32 \pi^{2}} \frac{1-r_{\Phi \mathrm{N}}+\ln r_{\Phi \mathrm{N}}}{\left(1-r_{\Phi \mathrm{N}}\right)^{2}} ; \\
\epsilon_{L}= & \frac{\left(z \mathcal{S}^{2} z^{T}\right)}{16 \pi^{2}} \frac{1-r_{\Phi \mathrm{N}}+r_{\Phi \mathrm{N}} \ln r_{\Phi \mathrm{N}}}{\left(1-r_{\Phi \mathrm{N}}\right)^{2}} ; \\
\epsilon_{H}= & -\frac{1}{8 \pi^{2}} \frac{m_{\mathrm{N}}}{v_{\mathrm{H}}}\left\{\left(z \mathcal{S}^{2} \mathcal{C} z^{T}\right) \frac{1-r_{\Phi \mathrm{N}}+r_{\Phi \mathrm{N}} \ln r_{\Phi \mathrm{N}}}{\left(r_{\Phi \mathrm{N}}-1\right)^{2}}\right. \\
& \left.+\frac{1}{2} \lambda_{x} \frac{v_{\mathrm{H}}^{2}}{m_{\mathrm{N}}^{2}}\left(z \mathcal{C} z^{T}\right) \frac{1-r_{\Phi \mathrm{N}}+\ln r_{\Phi \mathrm{N}}}{\left(r_{\Phi \mathrm{N}}-1\right)^{2}}\right\} .
\end{aligned}
$$




\section{B. DM self-interactions}

The self-interactions of the SIDM paradigm are generated in this model by $\Psi$ scattering mediated by $V$ exchange. There are two such reactions: $\Psi_{ \pm} \Psi_{ \pm} \rightarrow$ $\Psi_{ \pm} \Psi_{ \pm}$and $\Psi_{+} \Psi_{-} \rightarrow \Psi_{+} \Psi_{-}$, with cross section $\sigma_{r}$ and $\sigma_{a}$, respectively (the first is the same as Möller scattering with a massive photon). The calculation is straightforward, using

$$
\beta_{\Psi}=\sqrt{1-\frac{4 m_{\Psi}^{2}}{s}},
$$

and neglecting the DM mass difference, we find

$$
\begin{aligned}
\frac{\sigma_{x}}{m_{\Psi}}= & \frac{g_{\mathrm{V}}^{4}}{4 \pi s m_{\Psi}}\left\{\frac{\left(2 s+3 m_{\mathrm{V}}^{2}\right) s \beta_{\Psi}^{2}+2\left(m_{\mathrm{V}}^{2}+2 m_{\Psi}^{2}\right)^{2}}{2 m_{\mathrm{V}}^{2}\left(m_{\mathrm{V}}^{2}+s \beta_{\Psi}^{2}\right)}\right. \\
& \left.-\frac{\left(s \beta_{\Psi}^{2}+2 m_{\mathrm{V}}^{2}\right)\left(3 m_{\mathrm{V}}^{2}+4 m_{\Psi}^{2}\right)+2\left(m_{\mathrm{V}}^{2}+2 m_{\Psi}^{2}\right)^{2}-4 m_{\Psi}^{4}}{s \beta_{\Psi}^{2}\left(2 m_{\mathrm{V}}^{2}+s \beta_{\Psi}^{2}\right)} \ln \left(1+\frac{s \beta_{\Psi}^{2}}{m_{\mathrm{V}}^{2}}\right)\right\} ; \\
\frac{\sigma_{\mathrm{a}}}{m_{\Psi}}= & \frac{g_{\mathrm{V}}^{4}}{4 \pi s m_{\Psi}}\left\{\frac{\left(2 s+3 m_{\mathrm{V}}^{2}\right) s \beta_{\Psi}^{2}+2\left(m_{\mathrm{V}}^{2}+2 m_{\Psi}^{2}\right)^{2}}{2 m_{\mathrm{V}}^{2}\left(m_{\mathrm{V}}^{2}+s \beta_{\Psi}^{2}\right)}-\frac{\left(m_{\mathrm{V}}^{2}+s\right)}{s \beta_{\Psi}^{2}} \ln \left(1+\frac{s \beta_{\Psi}^{2}}{m_{\mathrm{V}}^{2}}\right)\right\} .
\end{aligned}
$$

These cross sections are enhanced when $m_{\Psi} \gg m_{\mathrm{V}}$ and the relative velocity $\beta_{\Psi}$ is small; in this regime the selfinteractions generated by $V$ meet the SIDM requirements.

Since $\Psi_{+}$and $\Psi_{-}$have a small mass difference, and have identical couplings, they will have the same relic abundance density $n$. In this case the effective DM-DM cross section will be $\left(\sigma_{r}+\sigma_{a}\right) / 2$. To see this, note that a $\Psi_{+}$ moving with speed $\mathrm{v}$; in a time $\delta t$ it will have $n \sigma_{r} \mathrm{v} \delta t$ interactions with other $\Psi_{+}$, and $n \sigma_{\mathrm{a}} \mathrm{\vee} \delta t$ interactions with the $\Psi_{-}$; the total number of interactions will be then (using $\left.n=n_{\mathrm{DM}} / 2\right)$,

$\frac{n_{\mathrm{DM}}}{2}\left(\sigma_{\mathrm{r}}+\sigma_{\mathrm{a}}\right) \mathrm{v} \delta t=n_{\mathrm{DM}} \sigma_{\mathrm{eff}} \mathrm{V} \delta t \Rightarrow \sigma_{\mathrm{eff}}=\frac{\sigma_{\mathrm{r}}+\sigma_{\mathrm{a}}}{2}$.

Note that $\sigma_{\text {eff }}$ depends on the relative velocity v.

Existing data constraints the SIDM cross section for galaxy clusters and for dwarf and low-surface-brightness galaxies; since the typical velocity in each environment is different, the cross section must have an appropriate velocity-dependence. The central values of the cross sections and velocities are [73]

$$
\begin{aligned}
\left.\frac{\sigma_{\text {eff }}}{m_{\Psi}}\right|_{\text {galaxy }} & =1.9 \frac{\mathrm{cm}^{2}}{\mathrm{gr}}, \\
\left.\frac{\sigma_{\text {eff }}}{m_{\Psi}}\right|_{\text {cluster }} & =0.1 \frac{\mathrm{cm}^{2}}{\mathrm{gr}} \\
\left.\beta_{\Psi}\right|_{\text {galaxy }} & =3.3 \times 10^{-4}, \\
\left.\beta_{\Psi}\right|_{\text {cluster }} & =5.4 \times 10^{-3} .
\end{aligned}
$$

Fitting Eqs. (13) and (14) to these values we find ${ }^{6}$

$$
m_{\mathrm{V}}=\frac{m_{\Psi}}{443}, \quad g_{\mathrm{V}}=\left(\frac{m_{\Psi}}{64 \mathrm{GeV}}\right)^{3 / 4} \text {. }
$$

\footnotetext{
${ }^{6}$ These relations imply $m_{\mathrm{V}} g_{\mathrm{V}}^{-4 / 3}=0.144 \mathrm{GeV}$, whose significance is unclear.
}

These expressions have significant errors; from [73] we estimate

$443 \rightarrow(116,1557), \quad 64 \mathrm{GeV} \rightarrow(17,225) \mathrm{GeV}$.

In our numerical calculations we will be conservative and assume that these are uncertain by up to a factor of 3 (e.g., that the first coefficient ranges from $443 / 3$ to $3 * 443$ ).

The DCC symmetry, despite being softly broken, is very effective in limiting the number of couplings of the $V$ that can have any phenomenological significance. For example, $V-Z$ and $V-\gamma$ mixings occur only at 2 and 3 loops, respectively, and can be ignored. The only interesting 1-loop vertex is considered in the next section.

\section{Decay of the $V$}

In the absence of the DCC breaking term $\propto \mu$ in Eq. (2), the massive dark photon $V$ is stable, which presents something of a problem: once it decouples from the $\Psi$, its abundance would be fixed, and since it is also light [cf. Eq. (16)], ${ }^{7}$ its presence would make the model inconsistent with big-bang nucleosynthesis (BBN) [74] and large scale structure formation (LSS) [75] constraints.

This is avoided when $\mu \neq 0$, that is, when the $\Psi_{ \pm}$mass degeneracy is broken. In this case, the graph in Fig. 2 generates an effective coupling of the form $\bar{n}_{L} V n_{L}$ that leads to

$$
\begin{aligned}
\Gamma(V & \left.\rightarrow \bar{n}_{L} n_{L}\right) \\
& =\frac{m_{\mathrm{V}}}{6 \pi}\left\{\frac{g_{\mathrm{V}}}{16 \pi^{2}}\left[f\left(\frac{m_{+}}{m_{\Phi}}\right)-f\left(\frac{m_{-}}{m_{\Phi}}\right)\right]\right\}^{2}\left(z \mathcal{S}^{2} z^{\dagger}\right)^{2},
\end{aligned}
$$

\footnotetext{
${ }^{7} m_{\Psi}<O(10 \mathrm{GeV})$ if we demand the absence of DM bound states. (Sec. II D), whence $m_{\mathrm{V}}$ will be in the $\mathrm{keV}$ range.
} 


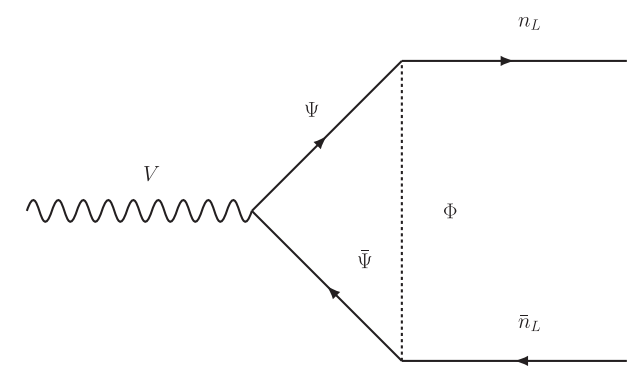

FIG. 2. Graphs responsible for a nonzero decay width for the $V$.

where $m_{ \pm}=m_{\Psi} \pm \mu$ denote the mass of $\Psi_{ \pm}$, we assumed $m_{\Psi} \gg m_{V}, \mu$, and defined

$$
f(x)=\frac{1}{4}\left(\frac{x^{2}+1}{x^{2}-1}\right)-\left(\frac{x^{2}}{x^{2}-1}\right)^{2} \ln x .
$$

The BBN and LSS constraints are relatively mild, $1 / \Gamma\left(V \rightarrow \bar{n}_{L} n_{L}\right)<1 \mathrm{~s}$, that we adopt in the numerical calculations.

\section{Bound states}

The inclusion of a self-interaction between DM particles opens up the possibility that the $\Psi_{+}$and $\Psi_{-}$, having opposite dark charges, will form bound states. If this were to happen the self-interactions would be screened and the cusp problem would reappear. To avoid this we now consider the conditions where such bound states do not form.

In the nonrelativistic limit, the $V$ exchange generates an attractive Yukawa potential between the $\Psi_{+}$and $\Psi_{-}$:

$$
\mathcal{V}_{N R}=\frac{g_{\mathrm{V}}^{2}}{4 \pi} \frac{e^{-m_{\mathrm{V}} r}}{r}
$$

If a bound state is formed then its typical size is determined by the range of the potential, $\sim 1 / m_{\mathrm{V}}$; it follows that the typical kinetic energy of the $\Psi$ will be $\sim m_{\mathrm{V}}^{2} / m_{\Psi}$, while their potential energy would be $\sim g_{\mathrm{V}}^{2} m_{\mathrm{V}} /(4 \pi)$. For the bound state to be unstable the kinetic energy must dominate: $g_{\mathrm{V}}^{2} m_{\mathrm{V}} /(4 \pi) \lesssim m_{\mathrm{V}}^{2} / m_{\Psi}$. These arguments are verified by exact calculations $[76,77]$ that give

$$
0.595 \frac{g_{\mathrm{V}}^{2}}{4 \pi}<\frac{m_{\mathrm{V}}}{m_{\Psi}}
$$

Using the values of $m_{\mathrm{V}}$ and $g$ obtained in Eq. (16) we find the following limit on $m_{\Psi}$ :

$$
m_{\Psi}<8.4 \mathrm{GeV}
$$

which is uncertain by up to a factor $\sim 6$.
Though $\left[\Psi_{+} \Psi_{-}\right]$bound states are allowed for larger $m_{\Psi}$, this does not necessarily imply that they will form. Formation occurs through the reactions $\Psi_{ \pm} \Psi_{+} \Psi_{-} \rightarrow$ $\left[\Psi_{+} \Psi_{-}\right] \Psi_{ \pm}$, with a virtual $V$ exchange, or $\Psi_{+} \Psi_{-} \rightarrow$ $\left[\Psi_{+} \Psi_{-}\right]+V$, with the (real or virtual) $V$ decaying subsequently to neutrinos. Calculating the rate for these reactions and determining the extent to which they affect the cusp problem in galactic DM distributions lies outside the scope of this paper. Here we will limit ourselves to the study of the model in the region $m_{\Psi}<10 \mathrm{GeV}$ where bound states do not form, and which is often outside the mass range considered in WIMP models (see, e.g., [78] and references therein). It is also worth noting that for these low masses the "neutrino floor" background in direct detection experiments rises by about 5 orders of magnitude (cf. 5); in Sec. VII we discuss to what extent this can conceal this model in this region of parameter space.

\section{ELECTROWEAK CONSTRAINTS}

In this section we summarize the constraints derived from high precision data on the invisible decay of the $Z$ and the Higgs, and from $W$-mediated meson decays; most of the results are the same as for an earlier simpler version of the model [48]. These effects are produced by the mixing (upon spontaneous symmetry breaking) of the Standard Model neutrino field with the mediators $\mathcal{F}$, which alters the couplings of the light mass eigenstates $n_{L}$ to the $W$ and $Z$, and introduces a coupling to the $H$ absent in the Standard Model.

\section{A. $Z$ invisible decay}

The addition of singlet Dirac fermions $N$ to the SM generate nonuniversal, though flavor diagonal, neutrino $(n)$ couplings to the $Z$ proportional to $\mathcal{C}^{2}$. In particular, the invisible $Z \rightarrow n n$ width will be proportional to $\operatorname{tr}\left(\mathcal{C}^{4}\right)$. The experimental value $\Gamma(Z \rightarrow$ inv $)=499.0 \pm 1.5 \mathrm{MeV}$ [79] for the invisible width of the $Z$ then generates a stringent bound on the parameters of the model when $m_{\mathrm{Z}}<m_{\mathrm{N}}$; if the $Z$ decays involving the $N$ are kinematically allowed, the constraints are somewhat weaker.

For the case of degenerate $N$ we find, using Eq. (8)

$$
\begin{aligned}
\Gamma(Z \rightarrow n n) & =\Gamma_{0} \operatorname{tr}\left\{\mathcal{C}^{4}\right\} ; \quad \Gamma_{0}=\left(\frac{g}{2 c_{\mathrm{W}}}\right)^{2} \frac{m_{\mathrm{Z}}}{24 \pi}, \\
\Gamma(Z \rightarrow N N) & =\Gamma_{0} \operatorname{tr}\left\{\mathcal{S}^{4}\right\}\left(1-r_{\mathrm{NZ}}\right) \sqrt{1-4 r_{\mathrm{NZ}}} \theta\left(1-4 r_{\mathrm{NZ}}\right), \\
\Gamma(Z \rightarrow N n) & =\Gamma_{0} \operatorname{tr}\left\{\mathcal{C}^{2} \mathcal{S}^{2}\right\}\left(2+r_{\mathrm{NZ}}\right)\left(1-r_{\mathrm{NZ}}\right)^{2} \theta\left(1-r_{\mathrm{NZ}}\right),
\end{aligned}
$$

so that the change in the invisible decay width of the $Z$ is given by 


$$
\begin{aligned}
& \frac{\Gamma(Z \rightarrow \text { inv })}{\Gamma_{\mathrm{SM}}(Z \rightarrow \text { inv })}-1 \\
& =\frac{1}{3}\left[-\operatorname{tr}\left\{\mathcal{S}^{2}\left(\mathbb{1}+\mathcal{C}^{2}\right)\right\}+\operatorname{tr}\left\{\mathcal{S}^{4}\right\}\left(1-r_{\mathrm{NZ}}\right)\right. \\
& \quad \times \sqrt{1-4 r_{\mathrm{NZ}}} \theta\left(1-4 r_{\mathrm{NZ}}\right) \\
& \left.\quad+\operatorname{tr}\left\{\mathcal{C}^{2} \mathcal{S}^{2}\right\}\left(2+r_{\mathrm{NZ}}\right)\left(1-r_{\mathrm{NZ}}\right)^{2} \theta\left(1-r_{\mathrm{NZ}}\right)\right] ;
\end{aligned}
$$

current experimental limits [79] require $\mid \Gamma(Z \rightarrow$ inv $) /$ $\Gamma_{\mathrm{SM}}(Z \rightarrow$ inv $)-1 \mid<0.0093$.

\section{B. $H$ invisible decays}

Using Eq. (8) we obtain (from Eqs. (10) and (11), $\Gamma(H \rightarrow \Psi \Psi) \propto \epsilon_{H}^{2}$ and is negligible $)$

$$
\begin{aligned}
\Gamma(H \rightarrow n, N)= & \frac{m_{\mathrm{H}}^{3}}{4 \pi v_{\mathrm{H}}^{2}}\left[r_{\mathrm{NZ}}\left(1-r_{\mathrm{NZ}}\right) \operatorname{tr}\left\{\mathcal{S}^{2} \mathcal{C}^{2}\right\} \theta\left(1-r_{\mathrm{NZ}}\right)\right. \\
& \left.+\frac{1}{2}\left(1-4 r_{\mathrm{NZ}}\right)^{3 / 2} \operatorname{tr}\left\{\mathcal{S}^{4}\right\} \theta\left(1-4 r_{\mathrm{NZ}}\right)\right], \\
\Gamma(H \rightarrow \Phi \Phi)= & \frac{\left(v_{\mathrm{H}} \lambda_{x}\right)^{2}}{16 \pi m_{\mathrm{H}}} \sqrt{1-4 r_{\Phi \mathrm{H}}} \theta\left(1-4 r_{\Phi \mathrm{H}}\right) .
\end{aligned}
$$

The total width of the $H$ is then $\Gamma(H)=\Gamma(H)_{\mathrm{SM}}+$ $\Gamma(H \rightarrow n, N)+\Gamma(H \rightarrow \Phi \Phi)$, with the SM contribution equal to $4.1 \mathrm{MeV}$ [80]; given that the limit on the invisible branching ratio is $25 \%[81,82]$, we find $\Gamma(H \rightarrow n, N)+$ $\Gamma(H \rightarrow \Phi \Phi)<1.26 \mathrm{MeV}$. Then, for degenerate $N$,

$$
\begin{aligned}
4.89 \times 10^{-4}> & \mid r_{\mathrm{NZ}}\left(1-r_{\mathrm{NZ}}\right) \operatorname{tr}\left\{\mathcal{S}^{2} \mathcal{C}^{2}\right\} \theta\left(1-r_{\mathrm{NZ}}\right) \\
& +\frac{1}{2}\left(1-4 r_{\mathrm{NZ}}\right)^{3 / 2} \operatorname{tr}\left\{\mathcal{S}^{4}\right\} \theta\left(1-4 r_{\mathrm{NZ}}\right) \\
& +1.93 \lambda_{x}^{2} \sqrt{1-4 r_{\Phi \mathrm{H}}} \theta\left(1-4 r_{\Phi \mathrm{H}}\right) \mid .
\end{aligned}
$$

\section{C. $W$-mediated decays}

The second line in Eq. (8) shows that charged current interactions of the leptons and the $W$ boson are also modified: using $r, s$ as flavor indices, the vertex involving a charged lepton $e_{L r}$ and a neutrino mass eigenstate $n_{L s}$ contains a factor $\left(V_{\mathrm{PMNS}}^{\dagger} \mathcal{C}\right)_{r s}$. This then implies (we assume that $m_{\mathrm{N}}>m_{\tau}$ )

$$
\begin{aligned}
\Gamma\left(\ell_{r} \rightarrow \ell_{s} \bar{\nu} \nu\right) & \simeq\left(1-\Delta_{r}-\Delta_{s}\right) \Gamma_{\mathrm{SM}}\left(\ell_{r} \rightarrow \ell_{s} \bar{\nu} \nu\right) ; \\
\Delta_{r} & =\left(V_{\mathrm{PMNS}}^{\dagger} \mathcal{S}^{2} V_{\mathrm{PMNS}}\right)_{r r}>0,
\end{aligned}
$$

(no sum over $r$ in the last expression). Note that the assumption $m_{\mathrm{N}}>m_{\tau}$ precludes the possibility of there being cancellations between the $n$ and $N$ contributions to these decays.
We define $R_{u \rightarrow X}=B(u \rightarrow X) / B_{\mathrm{SM}}(u \rightarrow X)-1$; then, for the specific decays of interest, we find (to $3 \sigma$ ),

$$
\begin{aligned}
R_{\tau \rightarrow \mu \nu \bar{\nu}} & \simeq B_{\mathrm{SM}}(\tau \rightarrow e \nu \bar{\nu}) \Delta_{e}-\left[1-B_{\mathrm{SM}}(\tau \rightarrow \mu \nu \bar{\nu})\right] \Delta_{\mu} \\
& \Rightarrow\left|0.8223 \Delta_{\mu}-0.1958 \Delta_{e}\right| \leq 0.0069, \\
R_{\tau \rightarrow e \nu \bar{\nu}} & \simeq B_{\mathrm{SM}}(\tau \rightarrow \mu \nu \bar{\nu}) \Delta_{\mu}-\left[1-B_{\mathrm{SM}}(\tau \rightarrow e \nu \bar{\nu})\right] \Delta_{e} \\
& \Rightarrow\left|0.1777 \Delta_{\mu}-0.8042 \Delta_{e}\right| \leq 0.0067, \\
R_{\pi \rightarrow \mu \nu} & \simeq B_{\mathrm{SM}}(\pi \rightarrow e \nu)\left(\Delta_{\mu}-\Delta_{e}\right) \Rightarrow\left|\Delta_{\mu}-\Delta_{e}\right| \leq 0.010 .
\end{aligned}
$$

We note that the limit derived from $\pi \rightarrow \mu e$ is not competitive: $\left|\Delta_{\mu}-\Delta_{e}\right| \leq 48.8$. Also, though the uncertainty in $\Gamma(\mu \rightarrow e \nu \bar{\nu})$ is very small, it does not lead to a constraint on $\Delta_{e}+\Delta_{\mu}$, since this decay is used as input data to fix the value of $G_{\mathrm{F}}$. One could use collider measurements of $m_{\mathrm{W}}$ and $g_{2}$ (the $S U\left(2_{L}\right)$ coupling constant in the SM) to predict this width, but the uncertainty is much larger and the limits are again not competitive.

\section{Lepton flavor violating processes: $\mu \rightarrow \boldsymbol{e} \gamma$}

In this model, the enW vertices differ from their SM counterparts (by a factor of $\mathcal{C}$ ); this, and the new $e N W$ vertices, generate a potentially important contribution to $\mu \rightarrow e \gamma$. A straightforward calculation gives

$$
\begin{aligned}
\Gamma(\mu \rightarrow e \gamma) & =\frac{\alpha m_{\mu}^{5}}{1024 \pi^{4} v_{\mathrm{H}}^{4}} f_{\mu}^{2}\left(r_{\mathrm{NW}}\right)|\delta|^{2} ; \\
\delta & =\left(V_{\mathrm{PMNS}}^{\dagger} \mathcal{S}^{2} V_{\mathrm{PMNS}}\right)_{12} \\
& =\sum_{i}\left(V_{\mathrm{PMNS}}\right)_{i 1}^{*} \mathcal{S}_{i}^{2}\left(V_{\mathrm{PMNS}}\right)_{i 2},
\end{aligned}
$$

where

$$
f_{\mu}(y)=\frac{(y-1)(y-3)(4 y-1)+2 y^{2}(5-2 y) \ln y}{2(y-1)^{4}} .
$$

The current limit on the branching ratio is $4.2 \times 10^{-13}$ [83]; this requires

$$
\left|f_{\mu}\left(r_{\mathrm{NW}}\right) \delta\right|<2.2 \times 10^{-5}
$$

\section{E. Muon anomalous magnetic moment}

The new $N N W$ vertices, and the $\mathcal{C}$ factors in the $n n W$ vertices in Eq. (8) generate nonstandard contributions to the anomalous magnetic moment of the muon, $a_{\mu}$. Using the results of [83] it is straightforward to see that

$$
\Delta a_{\mu}=\frac{G_{\mathrm{F}} m_{\mu}^{2}}{\sqrt{2} 8 \pi^{2}} \Delta_{\mu}\left[F\left(r_{\mathrm{NW}}\right)-F(0)\right]
$$




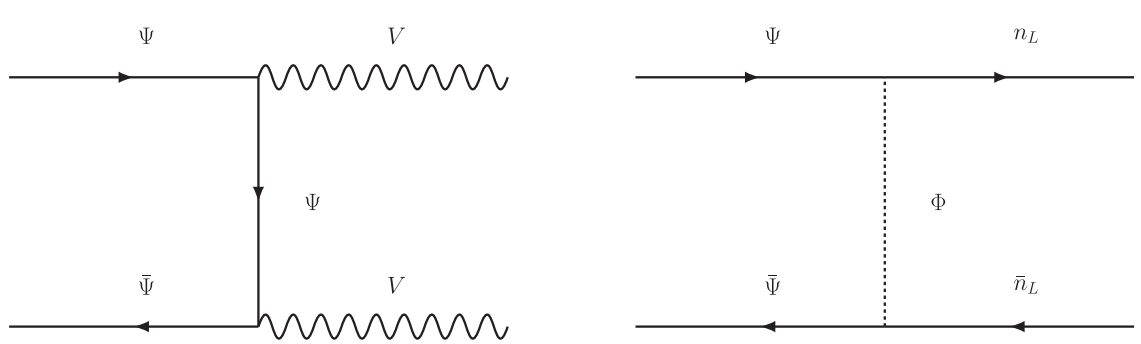

FIG. 3. Diagrams giving the leading contributions to the relic abundance cross sections.

where $\Delta_{\mu}=\Delta_{r=2}$ is defined in Eq. (27) and

$$
\begin{aligned}
F(w) & =\int_{0}^{1} d x \frac{2 x^{2}(1+x)+x(1-x)(2-x) w-x^{2}(x-1) k}{k x^{2}+(1-k) x+(1-x) w} ; \\
k & =\left(\frac{m_{\mu}}{m_{\mathrm{w}}}\right)^{2} \ll 1,
\end{aligned}
$$

so that

$F(w)-F(0) \simeq \frac{10-33 w+45 w^{2}-4 w^{3}}{6(1-w)^{3}}+\frac{3 w^{3} \ln w}{(1-w)^{4}}-\frac{5}{3}$,

and this ranges from 0 when $w=0$ to -1 when $w \rightarrow \infty$. Then

$$
\left|\Delta a_{\mu}\right| \leq \frac{G_{F} m_{\mu}^{2}}{\sqrt{2} 8 \pi^{2}} \Delta_{\mu}=1.17 \times 10^{-9} \Delta_{\mu}
$$

The constraints derived form $W$-mediated decays require $\Delta_{\mu} \lesssim 10^{-2}$ so $\left|\Delta a_{\mu}\right| \lesssim 10^{-11}$, while the current error [79] is $( \pm 5.4 \pm 3.3) \times 10^{-10}$. The anomalous magnetic moment limits do not produce a competitive bound now, but may do so with the upgraded Fermilab experiment [84]. ${ }^{8}$

\section{RELIC ABUNDANCE}

As the universe expands there will come a time when the $\Psi_{ \pm}$will cease to be in chemical equilibrium with the SM or with the dark photon sea. Still, we expect the interactions between $\Psi_{+}$and $\Psi_{-}$will keep them in equilibrium with each other and, since they have the same coupling to $V$ and approximately equal masses, they will have the same relic abundance; in the following we denote by $n_{\Psi}$ the total DM number density, adding the contributions from the $\Psi_{+}$ and $\Psi_{-}$.

The processes that determine the relic abundance are (see Fig. 3) $\Psi \bar{\Psi} \rightarrow n_{L} \bar{n}_{L}$ and $\Psi \bar{\Psi} \rightarrow V V$, with cross sections

$$
\begin{aligned}
\sigma_{\Psi \Psi \rightarrow n n} & =\frac{\left(z \mathcal{S}^{2} z^{T}\right)^{2}}{64 \pi s \beta_{\Psi}}\left[\frac{1+2 y(1+y)-\beta_{\Psi}^{2}}{(1+y)^{2}-\beta_{\Psi}^{2}}+\frac{y}{\beta_{\Psi}} \ln \left(\frac{1-\beta_{\Psi}+y}{1+\beta_{\Psi}-y}\right)\right], \\
\sigma_{\Psi \Psi \rightarrow \mathrm{VV}} & =\frac{g_{\mathrm{V}}^{4}}{8 \pi s} \frac{\beta_{\mathrm{V}}}{\beta_{\Psi}}\left[\frac{s m_{\Psi}^{2}+4\left(m_{\mathrm{V}}^{4}-2 m_{\mathrm{V}}^{2} m_{\Psi}^{2}-2 m_{\Psi}^{4}\right)}{s m_{\Psi}^{2}+m_{\mathrm{V}}^{2}\left(m_{\mathrm{V}}^{2}-4 m_{\Psi}^{2}\right)}+\frac{4\left(m_{\mathrm{V}}^{2}+m_{\Psi}^{2}\right)}{s \beta_{\mathrm{V}} \beta_{\Psi}} \ln \left|\frac{1+\beta_{\mathrm{V}}^{2}+2 \beta_{\Psi} \beta_{\mathrm{V}}}{1+\beta_{\mathrm{V}}^{2}-2 \beta_{\Psi} \beta_{\mathrm{V}}}\right|\right],
\end{aligned}
$$

where

$$
\begin{aligned}
y & =\frac{2\left(m_{\Phi}^{2}-m_{\Psi}^{2}\right)}{s} ; \quad \beta_{\Psi}=\sqrt{1-\frac{4 m_{\Psi}^{2}}{s}} ; \\
\beta_{\mathrm{V}} & =\sqrt{1-\frac{4 m_{\mathrm{V}}^{2}}{s}} .
\end{aligned}
$$

Since we are considering DM masses smaller than those for the $Z$ and $H$, there will be no resonant contributions to the relic abundance calculations, and the usual

\footnotetext{
${ }^{8}$ This model does not explain either the anomaly in the magnetic moment of the electron observed recently [85] since such effects will be suppressed by a factor $\left(m_{e} / m_{\mu}\right)^{2}$ with respect to the $(g-2)_{\mu}$.
}

approximations [86] can be reliably used. After a straightforward calculation we find

$$
\begin{aligned}
\left\langle\mathrm{v} \sigma_{\Psi \Psi \rightarrow n n}\right\rangle & \simeq \frac{\left(z \mathcal{S}^{2} z^{T}\right)^{2}}{32 \pi\left(r_{\Psi \Phi}+1\right)^{2} m_{\Psi}^{2}}, \\
\left\langle\mathrm{v} \sigma_{\Psi \Psi \rightarrow \mathrm{VV}}\right\rangle & \simeq \frac{g_{\mathrm{V}}^{4}}{16 \pi m_{\Psi}^{2}},
\end{aligned}
$$

where we summed over all final neutrino states and took $m_{\Psi} \gg m_{\mathrm{V}}$ [cf. Eq. (16)]; since there is no temperature dependence to lowest order, these are s-wave reactions.

We follow the usual prescription for the abundance calculation via the Boltzmann equation: 


$$
\frac{d n_{\Psi}}{d t}+3 H n_{\Psi}=-\sigma_{0}\left[n_{\Psi}^{2}-\left(n_{\Psi}^{(\mathrm{eq})}\right)^{2}\right]
$$

where $n_{\Psi}=n_{\Psi_{+}}+n_{\Psi_{-}}$, and ${ }^{9}$

$$
\begin{aligned}
\sigma_{0} & =\frac{1}{2}\left\langle\mathrm{v} \sigma_{\Psi \Psi \rightarrow n n}\right\rangle+\frac{1}{4}\left\langle\mathrm{v} \sigma_{\Psi \Psi \rightarrow \mathrm{VV}}\right\rangle \\
& =\frac{g_{\mathrm{V}}^{4}+\left[z \mathcal{S}^{2} z^{T} /\left(r_{\Psi \Phi}+1\right)\right]^{2}}{64 \pi m_{\Psi}^{2}} .
\end{aligned}
$$

Using the standard freeze-out approximation [86], the relic abundance $\Omega_{\Psi}$ is given by:

$$
\Omega_{\Psi} h^{2}=\frac{1.07 \times 10^{9}}{\mathrm{GeV}} \frac{x_{f}}{g_{\star} \xi} ; \quad \xi=\frac{M_{\mathrm{Pl}} \sigma_{0}}{\sqrt{g_{\star}}}
$$

where $M_{\mathrm{Pl}}$ denotes the Planck mass, $g_{\star \mathrm{s}}, g_{\star}$ denote, respectively, the relativistic degrees of freedom associated with the entropy and energy densities ${ }^{10}$ (for our case they are equal), and

$x_{f}=\frac{m_{\Psi}}{T_{f}}=\ln \left(0.076 m_{\Psi} \xi\right)-\frac{1}{2} \ln \left[\ln \left(0.076 m_{\Psi} \xi\right)\right]$,

with $T_{f}$ the freeze-out temperature. This expression for $\Omega_{\Psi}$ can now be compared to the result inferred from CMB data obtained by the Planck experiment $[88,89]$ :

$$
\Omega_{\text {Planck }} h^{2}=0.1186 \pm 0.006 \quad(3 \sigma),
$$

that constrains the parameters in Eq. (38). When the mixing angles $\mathcal{S}$ are small, as required by electroweak data, the presence of a dark-photon channel in Eq. (38) is essential for generating the experimentally required relic abundance.

\section{DIRECT DETECTION}

In the model under consideration the DM-nucleon scattering cross section responsible for a direct detection signal is generated by (t-channel) $Z$ and $H$ exchanges associated with the loop-induced couplings listed in Sec. II A. Since the momentum transfer is much smaller than $m_{\mathrm{Z}}$ and $m_{\mathrm{H}}$ we can approximate the relevant interaction by

$$
\begin{aligned}
\mathcal{L}_{\text {nucleon-DM }}= & \sqrt{2} G_{\mathrm{F}}\left[\bar{\Psi} \gamma_{\mu}\left(\epsilon_{L} P_{L}+\epsilon_{R} P_{R}\right) \Psi\right]\left(\overline{\mathfrak{p}} \mathcal{J}_{\mathfrak{p}}^{\mu} \mathfrak{p}\right. \\
& \left.+\overline{\mathfrak{n}} \mathcal{J}_{\mathfrak{n}}^{\mu} \mathfrak{n}\right)+G_{\mathrm{H}} \bar{\Psi} \Psi(\overline{\mathfrak{p}} \mathfrak{p}+\overline{\mathfrak{n}} \mathfrak{n}),
\end{aligned}
$$

where $\mathfrak{p}, \mathfrak{n}$ denote, respectively, the proton and neutron fields and ${ }^{11}$ [93]

$$
\begin{aligned}
& \mathcal{J}_{\mathfrak{p}}^{\mu}=\frac{1}{2}\left[\left(1-4 s_{\mathrm{W}}^{2}\right) \gamma^{\mu}+g_{A}\left(\gamma^{\mu}-\frac{2 m_{\mathcal{N}} q^{\mu}}{m_{\pi}^{2}+\mathbf{q}^{2}}\right) \gamma_{5}\right], \\
& \mathcal{J}_{\mathfrak{n}}^{\mu}=-\frac{1}{2}\left[\gamma^{\mu}+g_{A}\left(\gamma^{\mu}-\frac{2 m_{\mathcal{N}} q^{\mu}}{m_{\pi}^{2}+\mathbf{q}^{2}}\right) \gamma_{5}\right],
\end{aligned}
$$

with $m_{\mathcal{N}}, m_{\pi}$ the nucleon and pion masses, $\mathbf{q}$ the momentum transfer, $g_{A} \simeq-1.2723$ the axial nucleon coupling $[79,94]$

$$
G_{\mathrm{H}}=-\frac{0.011 \epsilon_{H}}{m_{\mathrm{H}}^{2}} .
$$

All isospin breaking effects in the Higgs-mediated interactions were ignored.

In the nonrelativistic limit this becomes

$$
\begin{aligned}
\left.\frac{1}{m_{\Psi} m_{\mathcal{N}}} \mathcal{L}_{\text {nucleon-DM }}\right|_{\mathrm{NR}}= & 4 G_{\mathrm{H}} \mathbb{1}_{\Psi} \mathbb{1}_{\mathcal{N}}+\sqrt{2} G_{\mathrm{F}}\left(\epsilon_{R}+\epsilon_{L}\right)\left\{\left[-2 s_{\mathrm{W}}^{2}+\left(1-2 s_{\mathrm{W}}^{2}\right)\right] \tau_{3}\right] \mathbb{1}_{\Psi} \mathbb{1}_{\mathcal{N}} \\
& \left.+\tau_{3}\left[\mathbf{s}_{\Psi} \cdot \mathbf{s}_{\mathcal{N}}-4 \frac{\left(\mathbf{q} \cdot \mathbf{s}_{\Psi}\right)\left(\mathbf{q} \cdot \mathbf{s}_{\mathcal{N}}\right)}{m_{\pi}^{2}+\mathbf{q}^{2}}\right]\left(\frac{\epsilon_{R}-\epsilon_{L}}{\epsilon_{R}+\epsilon_{L}}\right) g_{A}\right\}
\end{aligned}
$$

where $\tau_{3} \rightarrow 1$ for $\mathfrak{p}$ and $\tau_{3} \rightarrow-1$ for $\mathfrak{n}, \mathbf{S}_{\Psi, \mathcal{N}}$ and $\mathbb{1}_{\Psi, \mathcal{N}}$ denote the spin and unit (in spin space) operators for the DM and the nucleons, respectively. Using the notation and procedure described in [95,96] (see also [97]) we find that the DM-nucleus cross section, which we denote by $\sigma_{\mathcal{A}}$ is given by

$$
\begin{aligned}
\sigma_{\mathcal{A}}= & \frac{\left(m_{\mathcal{A}} / m_{\mathcal{N}}\right)^{2}}{16 \pi\left(m_{\mathcal{A}}+m_{\Psi}\right)^{2}}\left\{\kappa^{2}\left[(1+b)^{2} F_{M}^{(\mathfrak{p}, \mathfrak{p})}+(1-b)^{2} F_{M}^{(\mathfrak{n}, \mathfrak{n})}+2\left(1-b^{2}\right) F_{M}^{(\mathfrak{p}, \mathfrak{n})}\right]\right. \\
& \left.+\frac{\mathbb{K}^{2}\left(Q^{2}-2 Q+3\right)}{12}\left[F_{\Sigma^{\prime \prime}}^{(\mathfrak{p}, \mathfrak{p})}+F_{\Sigma^{\prime \prime}}^{(\mathfrak{n}, \mathfrak{n})}-2 F_{\Sigma^{\prime}}^{(\mathfrak{p}, \mathfrak{n})}+2\left(F_{\Sigma^{\prime}}^{(\mathfrak{p}, \mathfrak{p})}+F_{\Sigma^{\prime}}^{(\mathfrak{n}, \mathfrak{n})}-2 F_{\Sigma^{\prime}}^{(\mathfrak{p}, \mathfrak{n})}\right)\right]\right\},
\end{aligned}
$$

\footnotetext{
${ }^{9}$ The $1 / 2$ and $1 / 4$ factors account for the degeneracy of $\Psi_{ \pm}$in the processes in Fig. 3 that determine the total DM relic density $n_{\Psi}$. ${ }^{10}$ For our numerical calculations we use the expression of $g_{\star}$ in [87], not the one from [86].

${ }^{11}$ In the expressions for $\mathcal{J}_{\mathfrak{p}, \mathfrak{n}}^{\mu}$ we did not include a term $\propto \Delta s \gamma^{\mu} \gamma_{5}$ since the current experimental values for $\Delta s$ [90-92] are consistent with zero.
} 
where $\mathcal{A}$ is the atomic number, $m_{\mathcal{A}} \simeq \mathcal{A} m_{\mathcal{N}}$ the nuclear mass, and

$$
\begin{aligned}
\kappa & =\sqrt{2} G_{\mathrm{F}} m_{\Psi} m_{\mathcal{N}}\left[2\left(\epsilon_{L}+\epsilon_{R}\right) s_{\mathrm{W}}^{2}-2 \sqrt{2} \frac{G_{\mathrm{H}}}{G_{\mathrm{F}}}\right], \\
Q & =\frac{4|\mathbf{q}|^{2}}{|\mathbf{q}|^{2}+m_{\pi}^{2}}, \\
\mathbb{K} & =\frac{G_{\mathrm{F}}\left(\epsilon_{R}-\epsilon_{L}\right) m_{\Psi} m_{\mathcal{N}}}{\sqrt{2}} g_{A}, \\
b & =\frac{1-2 s_{\mathrm{W}}^{2}}{\sqrt{8} G_{\mathrm{H}} /\left[\left(\epsilon_{L}+\epsilon_{R}\right) G_{\mathrm{F}}\right]-2 s_{\mathrm{W}}^{2}} .
\end{aligned}
$$

The DM-nucleon cross section is then defined $[95,98]$ as

$$
\sigma_{N}=\left(\frac{m_{\mathcal{N}}}{m_{\mathcal{A}}}\right)^{2}\left(\frac{m_{\Psi}+m_{\mathcal{A}}}{m_{\Psi}+m_{\mathcal{N}}}\right)^{2} \frac{1}{\mathcal{A}^{2}} \sigma_{\mathcal{A}}
$$

If there are several isotopes, labeled by $I$, with abundances $\alpha_{I}$, then $F_{X}^{\left(N, N^{\prime}\right)} \rightarrow{ }^{I} F_{X}^{\left(N, N^{\prime}\right)}$ and

$$
\frac{1}{\mathcal{A}^{2}} F_{X}^{\left(N, N^{\prime}\right)} \rightarrow \sum_{I}{\frac{\alpha_{I}}{\mathcal{A}_{I}^{2}}}^{I} F_{X}^{\left(N, N^{\prime}\right)}=f_{X}^{\left(N, N^{\prime}\right)}
$$

so, defining

$$
\begin{aligned}
& f_{1}=f_{M}^{(\mathfrak{p}, \mathfrak{p})}+f_{M}^{(\mathfrak{n}, \mathfrak{n})}+2 f_{M}^{(\mathfrak{p}, \mathfrak{n})}, \\
& f_{2}=f_{M}^{(\mathfrak{p}, \mathfrak{p})}-f_{M}^{(\mathfrak{n}, \mathfrak{n})}, \\
& f_{3}=f_{M}^{(\mathfrak{p}, \mathfrak{p})}+f_{M}^{(\mathfrak{n}, \mathfrak{n})}-2 f_{M}^{(\mathfrak{p}, \mathfrak{n})}, \\
& f_{4}=\left(f_{\Sigma^{\prime \prime}}^{(\mathfrak{p}, \mathfrak{p})}+f_{\Sigma^{\prime \prime}}^{(\mathfrak{n}, \mathfrak{n})}-2 f_{\Sigma^{\prime \prime}}^{(\mathfrak{p}, \mathfrak{n})}\right)+\left(f_{\Sigma^{\prime}}^{(\mathfrak{p}, \mathfrak{p})}+f_{\Sigma^{\prime}}^{(\mathfrak{n}, \mathfrak{n})}-2 f_{\Sigma^{\prime}}^{(\mathfrak{p}, \mathfrak{n})}\right),
\end{aligned}
$$

the expression for the DM-nucleon cross section takes the relatively simple form

$$
\sigma_{\mathcal{N}}=\frac{1}{16 \pi^{2}\left(m_{\mathcal{N}}+m_{\Psi}\right)^{2}}\left[\left(f_{1}+2 b f_{2}+b^{2} f_{3}\right) \kappa^{2}+\frac{\mathbb{K}^{2}\left(Q^{2}-2 Q+3\right)}{12} f_{4}\right] .
$$

It is worth noting that the term $\propto \kappa^{2}$ is the spin-independent contribution, while that $\propto \mathbb{K}^{2}$ is the spin-dependent one. The expected suppression of the latter with respect to the former follows from $f_{4} \ll f_{1}$. In the calculations we use the expressions for the ${ }^{I} F_{X}^{\left(N, N^{\prime}\right)}$ provided in [95] for Xe and $\mathrm{Ge}$, and in [99] ${ }^{12}$ for $\mathrm{CaWO}_{4}$ :

\begin{tabular}{c|c|c|c|c} 
material & $f_{1}$ & $f_{2}$ & $f_{3}$ & $f_{4} \times 10^{4}$ \\
\hline $\mathrm{Xe}$ & $0.995256-6.98794 \mathbf{q}^{2}$ & $-0.177925+1.39348 \mathbf{q}^{2}$ & $0.031717-0.314739 \mathbf{q}^{2}$ & $0.142261-1.22925 \mathbf{q}^{2}$ \\
$\mathrm{Ge}$ & $0.990137-6.97097 \mathbf{q}^{2}$ & $-0.124142+0.960981 \mathbf{q}^{2}$ & $0.0161359-0.115939 \mathbf{q}^{2}$ & $0.156404-1.61629 \mathbf{q}^{2}$ \\
$\mathrm{CaWO}_{4}$ & $0.0624983-0.447775 \mathbf{q}^{2}$ & 0 & 0 & 0
\end{tabular}

and we took $|\mathbf{q}|=m_{\Psi} \times 300 \mathrm{~km} / \mathrm{s}$.

We note that the dependence of $\sigma_{\mathcal{N}}$ on $m_{\Psi}$ is simple and contained in the factor $\left[m_{\Psi} /\left(m_{\Psi}+m_{\mathcal{N}}\right)\right]^{2}$, it also has a more complicated dependence on $m_{\Phi}, m_{\mathrm{N}}$ through the parameters $\epsilon_{L, R, H}$.

In the numerical results below we used the experimental constraints on the direct detection cross section published by Xenon1T [100], PandaX [101], CDMS [102] and CRESST [103] for the range $0.36 \mathrm{GeV} \leq$ $m_{\Psi} \leq 10 \mathrm{GeV}$; in cases where the mass ranges of two experiments overlap we take the strictest limit. Specifically, we used:

\footnotetext{
${ }^{12}$ Note that there is a normalization factor of $\pi$ difference between the conventions of [95,99]; for example $F_{M}^{(\mathfrak{p}, \mathfrak{p})}$ in [95] equals $\pi \times\left[W_{M}^{(0,0)}+2 W_{M}^{(0,1)}+W_{M}^{(1,1)}\right]$ in [99].
}

\begin{tabular}{c|c} 
Experiment & $m_{\Psi}$ range $(\mathrm{GeV})$ \\
\hline Xenon1T & $(6.06,10.0)$ \\
PandaX & $(4.12,6.06)$ \\
CDMS & $(1.61,4.12)$ \\
CRESST & $(0.36,1.61)$
\end{tabular}

as illustrated in Fig. 5.

\section{INDIRECT DETECTION}

As DM accumulates at the center of astrophysical objects and thermalizes [104], it can annihilate into SM particles and, in principle, produce an observable signal. The most distinctive of such indirect detection signatures is the annihilation of $\Psi$ 's into neutrinos, which produces a monochromatic neutrino line, a signal expected from both the sun and the galactic halo. There are also additional channels through the $Z$ and $H$ bosons, but the corresponding 
couplings are small, since they are generated at one loop (Sec. II A). For the range of masses being considered, the neutrino signal is obscured by the much larger atmospheric neutrino background [105-107], so its detection will be difficult with Earth-based neutrino observatories. The cross sections for the other channels also lie significantly below the existing experimental sensitivities. The quantitative results are the same as in the absence of self-interactions [48] and will not be reproduced here for that reason.

The existence of dark scalar $\Phi$ can be probed in principle by accurate measurements of the cosmological or astrophysical neutrino flux, since the scattering of high-energy neutrinos off the ambient DM will exhibit a resonance ${ }^{13}$ at neutrino energy $E_{\nu}^{(\text {res })}=\left(m_{\Phi}^{2}-m_{\Psi}^{2}\right) /\left(2 m_{\Psi}\right)$; numerically, $E_{\nu}^{(\text {res })} \sim 1 \mathrm{GeV}$ to $3.7 \mathrm{TeV}$ (or $\sim 1$ to $30 \mathrm{GeV}$ in the small $z$ scenario), roughly independent of $m_{\Psi}$. Observation of this effect is also challenging because of the significant background from the atmospheric neutrino flux at these energies. The dark photons influence the relic abundance and, being long lived, may have effects detectable by the nextgeneration $\mathrm{CMB}$ experiments; possible effects of these decays will be explored in a future publication.

\section{NUMERICAL RESULTS}

As we mention before, the model being considered has in total 11 free parameters: $m_{\Psi}, \mu, m_{\Phi}, m_{\mathbb{N}}, \lambda_{x}, \mathcal{S}$, and $z$ (assuming $m_{\mathrm{V}}$ and $g$ are fixed by Eqs. (16) and (17) and taking $z$ is real). In this section we consider the region in parameter space

$$
\begin{aligned}
& 0.5 \mathrm{GeV} \leq m_{\Psi} \leq 10 \mathrm{GeV}, \quad \mu=\frac{m_{\Psi}}{20}, \\
& \min \left\{1.1 m_{\Psi}, m_{\Psi}+2 \mathrm{GeV}\right\} \leq m_{\Phi}<500 \mathrm{GeV}, \\
& \min \left\{1.1 m_{\Psi}, m_{\Psi}+2 \mathrm{GeV}\right\} \leq m_{\mathrm{N}} \leq 1.5 \mathrm{TeV}, \\
& \left|S_{i}\right|<1, \quad\left|\lambda_{x}\right| \leq \pi, \quad\left|z_{i}\right|^{2} \leq 10 \text { or } 1 \quad(i=1,2,3) ;
\end{aligned}
$$

and determine the subregion allowed by the various constraints listed above. This is frequently carried out by reducing the number of free parameters (e.g., fixing the $\mathcal{S}$ and taking all the $z_{i}$ equal [48]) and then doing a uniform scan in the reduced space. Here we follow a different route: we do not adopt any simplifying relations between the parameters (except $\mu$ ), and concentrate on finding the boundary of the allowed subregion; this then becomes a non-linear optimization problem that can be treated using standard techniques [110]. In our calculations we use a publicly available nonlinear programming package NLopt [111].

The upper bound for $m_{\Psi}$ is motivated by requiring the absence of the bound states (Sec. II D), while the lower bound corresponds roughly to the lowest value probed by

\footnotetext{
${ }^{13}$ This effect is similar to the scattering of high-energy neutrinos off the cosmic neutrino background [108,109].
}

direct-detection experiments (Sec. V). The lower value of $m_{\Phi}$ ensures the dark scalar is heavier than the fermions; the upper bound is arbitrary. The lower value of $m_{\mathrm{N}}$ are set to avoid co-annihilation channels and ensure $m_{\mathrm{N}}>m_{\tau}$ as assumed in Sec. III C; the upper bound is also arbitrary. The range of the $\mathcal{S}_{i}$ it the one allowed by Eq. (6). The limit on $\lambda_{x}$ is motivated by perturbativity. We also consider two scenarios where the maximum value of the Yukawa couplings $|z|^{2}$ is large $(=10)$ or small $(=1)$. Finally, the mass split $\mu$ is chosen to ensure the DCC breaking is weak; the results are independent of this choice as long as $\mu \ll m_{\Psi}$.

We define

$$
\langle\operatorname{mix}\rangle=\sum\left|z_{i}\right|^{2} \mathcal{S}_{i}^{2}, \quad\langle z\rangle=|z| / \sqrt{3},
$$

as measures of the mixing strength and Yukawa coupling of the mediators, and then obtain the projections of the allowed sub-region in the $m_{\Psi}-m_{\Phi}, m_{\Psi}-\lambda_{x}, m_{\Psi}-\langle z\rangle$ and the $m_{\Psi}-\langle\operatorname{mix}\rangle$ planes. The results are presented in Figs. 4 $(a)-(d)$ respectively. In the $m_{\Psi}-m_{\mathrm{N}}$ plane the constraints allow the full range indicated in Eq. (54); that is, for each $m_{\mathrm{N}}$ in this range there are values of the other parameters for which all constraints are satisfied (in general these values change for each choice of $m_{\Psi}$ and $m_{\mathrm{N}}$ ). The figures also indicate the differences between the large $\left|z_{i}\right| \leq 10$ and small $\left|z_{i}\right| \leq 1$ cases, and the effects of the projected direct detection constraints form the SCMDS detector.

Of the several constraints the ones derived from the $Z$ and $H$ invisible widths, the $W$ mediated decays and $\mu \rightarrow e \gamma$ (Sec. III A-III D) require small mixing angles $\mathcal{S}_{i}^{2} \lesssim 0.1$, as show in Fig. 4(d). The relic abundance constraint (Sec. IV) requires $\sigma_{0}$ in Eq. (38) to be about $1 \%$ of a typical electroweak cross section, which requires $g_{\mathrm{V}}$ in the upper values of its allowed range defined by Eqs. (16) and (17). Finally, the direct detection constraint requires $\left(z^{T} z\right) \lambda_{x}$ and $\left(z^{T} \mathcal{S}^{2} z\right)$ to be small, while the $V$ lifetime requires $\left(z^{T} \mathcal{S}^{2} z\right)\left(\mu m_{\Psi} / m_{\Phi}^{2}\right)$ to relatively large, which excludes very small values of $z$ [Fig. 4(c)], large values of $m_{\Phi}$ [Fig. 4(a)], and all but small values of the Higgs portal coupling $\left|\lambda_{x}\right|$ when $\left|z_{i}\right| \leq 1$ or $m_{\Psi} \lesssim 0.5 \mathrm{GeV}$ [Fig. 4(b)]. That there are large allowed regions of parameter space is due to the symmetries of the model, and our reliance on the neutrino portal, conditions that naturally suppress the direct and indirect detection signals (the latter due to the very small mixing with the photon). Nonetheless the data do require some constraints, excluding large values of $m_{\Phi}$ and of the $\mathcal{S}_{i}$, and, for small $m_{\Psi}$, large values of $\left|\lambda_{x}\right|$.

In Fig. 5 we plot the values of the direct-detection cross sections for a selection of points on or close to the boundary of the allowed region of parameter space. The points are chosen only to illustrate that there is a region of parameter space within the sensitivity reach of SuperCDMS [112], but that this experiment cannot exclude the model; it is also worth noting that a (larger) region of parameter space will 


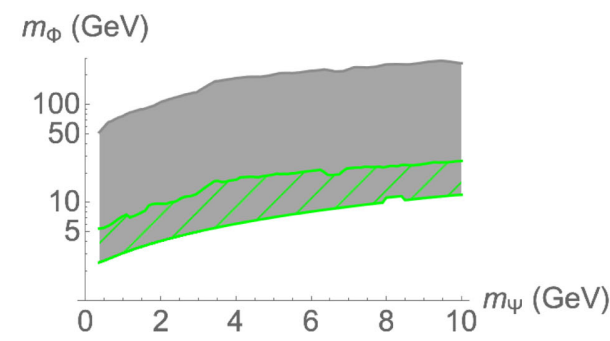

(a)

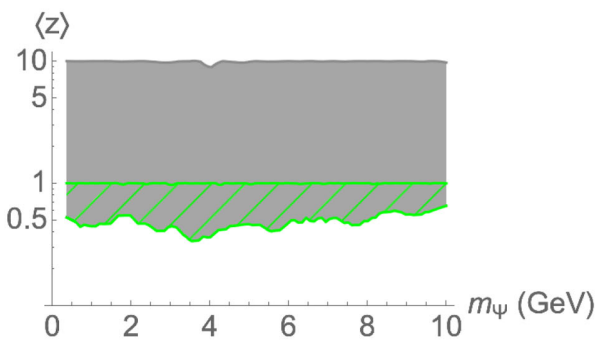

(c)

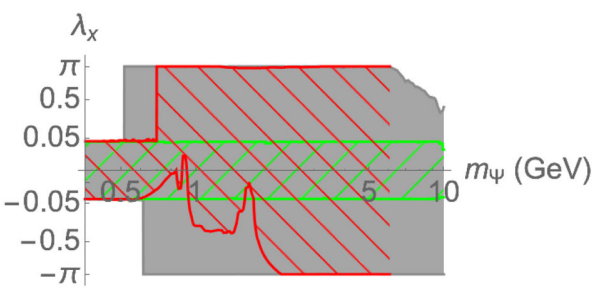

(b)

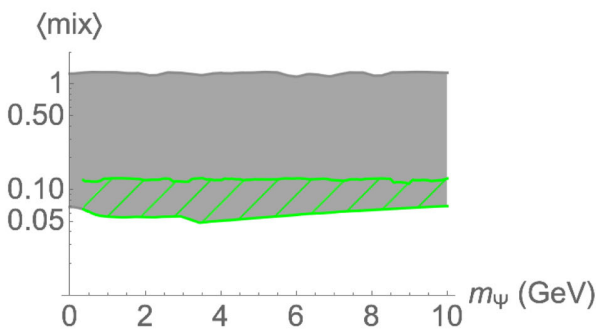

(d)

FIG. 4. Projections of the allowed parameter region when large values of $z,\left|z_{i}\right|^{2} \leq 10$, are allowed (cf. Eq. (54) and text), (a) in the $m_{\Psi}-m_{\Phi}$ plane, $(b)$ the $m_{\Psi}-\lambda_{x}$ plane, $(c)$ the $m_{\Psi}-\langle z\rangle$ plane (where $\langle z\rangle=|z| / \sqrt{3}$ ), and $(d)$ the $m_{\Psi}-\langle\operatorname{mix}\rangle$ plane, where $\langle$ mix $\rangle$ is defined in Eq. (55). The red hatched area in (b) gives the region that would be allowed by SCDMS for $m_{\Psi} \leq 6 \mathrm{GeV}$ (for larger masses XENON already provides stricter bounds); the green hatched areas correspond to smaller allowed values of $z,\left|z_{i}\right| \leq 1$. The unevennesses in the curves are due to numerical inaccuracies.

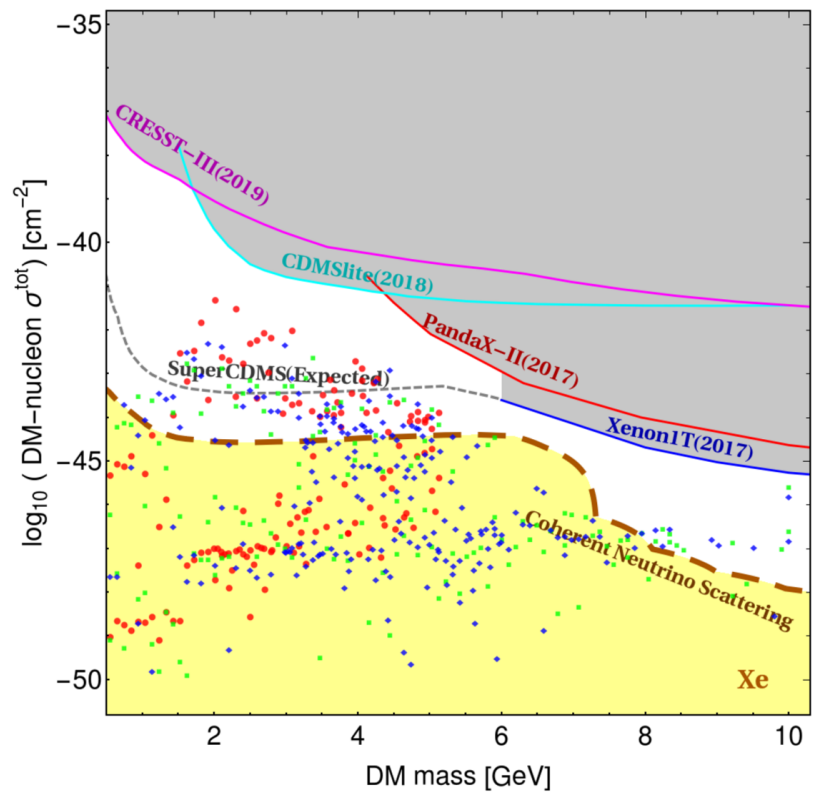

FIG. 5. Experimental limits on the direct detection cross section $\sigma$. The upper curves are obtained, from left to right, from the CRESST, CDMS, PandaX and Xenon1T experiments, and the expected sensitivity limit for the superCDMS experiment [112]; the coherent neutrino scattering regions are calculated for Xe [113]. For illustration we also include the cross sections corresponding to a selection of points on the boundary of the allowed region of parameter space, on the upper and lower boundaries of Fig. 4(a) (green points), of Fig. 4(c) (red points), and of Fig. 4(d) (blue points). correspond to cross sections above the coherent neutrino scattering "floor" [113]. Both these regions are significant in size: restricting the model to either (or both) would not require fine tuning.

\section{CONCLUSIONS}

In this paper, we have considered an extension of the neutrino-portal DM scenario, introducing self-interactions to the dark sector via a $U(1)_{\text {dark }}$ local symmetry with its corresponding vector boson $V$. The dark sector consists of a scalar $\Phi$, the dark photon $V$, and two almost degenerate fermions $\Psi_{ \pm}$, of opposite $U(1)_{\text {dark }}$ charges and which constitute the DM relics. We have also imposed a (softly broken) $\mathbb{Z}_{2}$ dark-charge symmetry that strongly suppresses $V$ mixings with the SM photon and $Z$, but still allows for the $V$ to decay into neutrinos with a sufficiently short lifetime, as required by phenomenology; in addition, the dark scalar has a natural coupling to the SM Higgs doublet. The model then also contains a Higgs portal and a naturally suppressed vector portal to the neutrino sector, each dominating in a different region of parameter space, though in this publication we concentrated on situations where the neutrino portal is more significant.

The direct and indirect detection cross sections are naturally suppressed while the annihilation cross section is adequately large without fine-tuning; these features are mostly responsible for the weak restrictions on the model parameters required by current data (more than the 
relatively large number of parameters in the model). Though this avoids the most stringent constraints, other experimental and observational data impose restrictions on parameter space. Electroweak precision data (Sec. III AIII D) restrict the mixing angles $\mathcal{S}$ to small values. The relic abundance constraint is sensitive to the dark gauge coupling $g_{\mathrm{V}}$ [cf. Eqs. (38) to (40)], with larger values [see Eqs. (16) and (17)] disfavored since the lead to DM underproduction; a more precise determination of the DM cross section as a function of velocity will provide a strict test of the viability of this model. The core-cusp data in galaxies and clusters place limits on the dark photon coupling and mass, albeit with significant errors.

As discussed in Sec. VII, the interplay between the direct detection and $V$ lifetime constraints can provide important parameter restrictions, depending on the allowed range of values for the Yukawa couplings $z$. Due to the very suppressed mixing of the $V$ with the photon, constraints derived from stellar cooling (through $V \rightarrow \nu \nu$ ) are not important. Indirect detection limits do not generate significant constraints on the model; this is because these limits are relatively weak for neutrinos, while for all other final states the cross sections in this model are very small. We have not included constraints derived from neutrino oscillations because they are not precise enough to provide significant limits. The same applies to existing limits derived from the measurement of the muon anomalous magnetic moment, in this case, however, an improvement by one order of magnitude in the experimental sensitivity would provide useful constraints on this model (though only on the mixing angles $\mathcal{S}$ ). Overall, current data allows large regions of parameter space; new experiments (e.g., SCDMS) are expected to provide modest additional restrictions [cf. Fig. 4(b)].

The presence of oppositely charged DM components may allow the formation of bound states; if this occurs, and if the formation rate of such bound states is sufficiently high, the cusp-core problem would reappear as the interactions between the bound states will be weak (akin to the Van der Waals interactions). In this paper we took a conservative approach and simply required that the potential generated by the $V$ should not lead to $\left[\Psi_{+} \Psi_{-}\right]$bound states (by assuming that these particles are sufficiently light); we will return to the issue of bound state formation in a future publication.

\section{ACKNOWLEDGMENTS}

This work has been supported by the University of California Institute for Mexico and the United States (UC MEXUS) (CN 18-128) and the Consejo Nacional de Ciencia y Tecnología (CONACYT) (CN 18-128). The authors would like to thank Hai-Bo Yu for interesting and useful comments. J. M. L. acknowledges the University of California, Riverside, for its warm hospitality.
[1] F. Zwicky, Helv. Phys. Acta 6, 110 (1933); Gen. Relativ. Gravit. 41, 207 (2009).

[2] V. C. Rubin and W. K. Ford, Jr., Astrophys. J. 159, 379 (1970).

[3] E. Corbelli and P. Salucci, Mon. Not. R. Astron. Soc. 311, 441 (2000).

[4] S. W. Allen, A. E. Evrard, and A. B. Mantz, Annu. Rev. Astron. Astrophys. 49, 409 (2011).

[5] D. Clowe, M. Bradac, A. H. Gonzalez, M. Markevitch, S. W. Randall, C. Jones, and D. Zaritsky, Astrophys. J. 648, L109 (2006).

[6] S. Hanany et al. (NASA PICO Collaboration), arXiv:1902 .10541.

[7] E. Behnke et al., Astropart. Phys. 90, 85 (2017).

[8] C. Fu et al. (PandaX-II Collaboration), Phys. Rev. Lett. 118, 071301 (2017); 120, 049902(E) (2018).

[9] D. S. Akerib et al. (LUX Collaboration), Phys. Rev. Lett. 116, 161302 (2016).

[10] E. Aprile et al. (XENON Collaboration), Phys. Rev. Lett. 119, 181301 (2017).

[11] D. S. Akerib et al. (LUX Collaboration), Phys. Rev. Lett. 118, 021303 (2017).

[12] A. Tan et al. (PandaX-II Collaboration), Phys. Rev. Lett. 117, 121303 (2016).
[13] E. Bulbul, M. Markevitch, A. Foster, R. K. Smith, M. Loewenstein, and S. W. Randall, Astrophys. J. 789, 13 (2014).

[14] O. Ruchayskiy, A. Boyarsky, D. Iakubovskyi, E. Bulbul, D. Eckert, J. Franse, D. Malyshev, M. Markevitch, and A. Neronov, Mon. Not. R. Astron. Soc. 460, 1390 (2016).

[15] J. Franse et al., Astrophys. J. 829, 124 (2016).

[16] O. Urban, N. Werner, S. W. Allen, A. Simionescu, J. S. Kaastra, and L. E. Strigari, Mon. Not. R. Astron. Soc. 451, 2447 (2015).

[17] F. A. Aharonian et al. (Hitomi Collaboration), Astrophys. J. 837, L15 (2017).

[18] K. Choi et al. (Super-Kamiokande Collaboration), Phys. Rev. Lett. 114, 141301 (2015).

[19] M. G. Aartsen et al. (IceCube Collaboration), Eur. Phys. J. C 77, 146 (2017); 79, 214(E) (2019).

[20] M. Ackermann et al. (Fermi-LAT Collaboration), Astrophys. J. 840, 43 (2017).

[21] D. Hooper and L. Goodenough, Phys. Lett. B 697, 412 (2011).

[22] M. Ackermann et al. (Fermi-LAT Collaboration), Phys. Rev. Lett. 115, 231301 (2015).

[23] M.-Y. Cui, Q. Yuan, Y.-L. S. Tsai, and Y.-Z. Fan, Phys. Rev. Lett. 118, 191101 (2017). 
[24] M. Aaboud et al. (ATLAS Collaboration), J. High Energy Phys. 09 (2016) 175.

[25] A. M. Sirunyan et al. (CMS Collaboration), J. High Energy Phys. 06 (2018) 027.

[26] D. Abercrombie et al., Phys. Dark Universe 27, 100371 (2020).

[27] W. J. G. de Blok, Adv. Astron. 2010, 789293 (2010).

[28] R. A. Flores and J. R. Primack, Astrophys. J. 427, L1 (1994).

[29] B. Moore, Nature (London) 370, 629 (1994).

[30] B. Moore, T. R. Quinn, F. Governato, J. Stadel, and G. Lake, Mon. Not. R. Astron. Soc. 310, 1147 (1999).

[31] M. G. Walker and J. Penarrubia, Astrophys. J. 742, 20 (2011).

[32] S.-H. Oh, C. Brook, F. Governato, E. Brinks, L. Mayer, W. J. G. de Blok, A. Brooks, and F. Walter, Astron. J. 142, 24 (2011).

[33] J. S. Bullock and M. Boylan-Kolchin, Annu. Rev. Astron. Astrophys. 55, 343 (2017).

[34] A. A. Klypin, A. V. Kravtsov, O. Valenzuela, and F. Prada, Astrophys. J. 522, 82 (1999).

[35] B. Moore, S. Ghigna, F. Governato, G. Lake, T. R. Quinn, J. Stadel, and P. Tozzi, Astrophys. J. 524, L19 (1999).

[36] M. Boylan-Kolchin, J. S. Bullock, and M. Kaplinghat, Mon. Not. R. Astron. Soc. 415, L40L44 (2011).

[37] M. Boylan-Kolchin, J. S. Bullock, and M. Kaplinghat, Mon. Not. R. Astron. Soc. 422, 1203 (2012).

[38] S. Garrison-Kimmel, M. Boylan-Kolchin, J. S. Bullock, and E. N. Kirby, Mon. Not. R. Astron. Soc. 444, 222 (2014).

[39] E. Papastergis, R. Giovanelli, M. P. Haynes, and F. Shankar, Astron. Astrophys. 574, A113 (2015).

[40] K. A. Oman, A. Marasco, J. F. Navarro, C. S. Frenk, J. Schaye, and A. Bentez-Llambay, Mon. Not. R. Astron. Soc. 482, 821 (2019).

[41] G. Rhee, A. Klypin, and O. Valenzuela, Astrophys. J. 617, 1059 (2004).

[42] W. J. G. d. Blok, A. Bosma, and S. S. McGaugh, Mon. Not. R. Astron. Soc. 340, 657 (2003).

[43] A. Genina, A. Bentez-Llambay, C. S. Frenk, S. Cole, A. Fattahi, J. F. Navarro, K. A. Oman, T. Sawala, and T. Theuns, Mon. Not. R. Astron. Soc. 474, 13981411 (2017).

[44] J. I. Read, M. G. Walker, and P. Steger, Mon. Not. R. Astron. Soc. 481, 860 (2018).

[45] J. Zavala and C. S. Frenk, Galaxies 7, 81 (2019).

[46] S. Tulin and H.-B. Yu, Phys. Rep. 730, 1 (2018).

[47] D. N. Spergel and P. J. Steinhardt, Phys. Rev. Lett. 84, 3760 (2000).

[48] V. González-Macías, J. I. Illana, and J. Wudka, J. High Energy Phys. 05 (2016) 171.

[49] V. González-Macías, J. Illana, and J. Wudka, J. Phys. Conf. Ser. 761, 012082 (2016).

[50] N. Cosme, L. L. Honorez, and M. H. G. Tytgat, Phys. Rev. D 72, 043505 (2005).

[51] A. Falkowski, J. Juknevich, and J. Shelton, arXiv:0908 .1790 .

[52] H. An, S.-L. Chen, R. N. Mohapatra, and Y. Zhang, J. High Energy Phys. 03 (2010) 124.

[53] M. Lindner, A. Merle, and V. Niro, Phys. Rev. D 82, 123529 (2010).
[54] A. Falkowski, J. T. Ruderman, and T. Volansky, J. High Energy Phys. 05 (2011) 106.

[55] Y. Farzan, J. High Energy Phys. 02 (2012) 091.

[56] J. Heeck and H. Zhang, J. High Energy Phys. 05 (2013) 164.

[57] S. Baek, P. Ko, and W.-I. Park, J. High Energy Phys. 07 (2013) 013.

[58] I. Baldes, N. F. Bell, A. J. Millar, and R. R. Volkas, J. Cosmol. Astropart. Phys. 10 (2015) 048.

[59] M. Escudero, N. Rius, and V. Sanz, J. High Energy Phys. 02 (2017) 045.

[60] M. Escudero, N. Rius, and V. Sanz, Eur. Phys. J. C 77, 397 (2017).

[61] B. Batell, T. Han, and B. S. E. Haghi, Phys. Rev. D 97, 095020 (2018).

[62] S. HajiSadeghi, S. Smolenski, and J. Wudka, Phys. Rev. D 99, 023514 (2019).

[63] A. Berlin and N. Blinov, Phys. Rev. D 99, 095030 (2019).

[64] P. Bandyopadhyay, E. J. Chun, R. Mandal, and F. S. Queiroz, Phys. Lett. B 788, 530 (2019).

[65] M. Blennow, E. Fernandez-Martinez, A. O.-D. Campo, S. Pascoli, S. Rosauro-Alcaraz, and A. V. Titov, Eur. Phys. J. C 79, 555 (2019).

[66] B. Holdom, Phys. Lett. 166B, 196 (1986).

[67] J. Alexander et al., arXiv:1608.08632.

[68] E. Ma, Phys. Lett. B 772, 442 (2017).

[69] M. Duerr, K. Schmidt-Hoberg, and S. Wild, J. Cosmol. Astropart. Phys. 09 (2018) 033.

[70] D. Wyler and L. Wolfenstein, Nucl. Phys. B218, 205 (1983).

[71] R. N. Mohapatra and J. W. F. Valle, Phys. Rev. D 34, 1642 (1986).

[72] E. Ma, Phys. Lett. B 191, 287 (1987).

[73] M. Kaplinghat, S. Tulin, and H.-B. Yu, Phys. Rev. Lett. 116, 041302 (2016).

[74] B. Ahlgren, T. Ohlsson, and S. Zhou, Phys. Rev. Lett. 111, 199001 (2013).

[75] Y. Zhang, J. Cosmol. Astropart. Phys. 05 (2015) 008.

[76] H. An, M. B. Wise, and Y. Zhang, Phys. Rev. D 93, 115020 (2016).

[77] J. P. Edwards, U. Gerber, C. Schubert, M. A. Trejo, and A. Weber, Prog. Theor. Exp. Phys. 2017 (2017), 10.1093/ ptep/ptx107.

[78] H. Baer, K.-Y. Choi, J. E. Kim, and L. Roszkowski, Phys. Rep. 555, 1 (2015).

[79] M. Tanabashi et al. (Particle Data Group), Phys. Rev. D 98, 030001 (2018).

[80] D. de Florian et al. (LHC Higgs Cross Section Working Group), CERN Report No ERN-2017-002-M, arXiv:1610 .07922 .

[81] M. Aaboud et al. (ATLAS Collaboration), Phys. Rev. Lett. 122, 231801 (2019).

[82] A. M. Sirunyan et al. (CMS Collaboration), Phys. Lett. B 793, 520 (2019).

[83] J. P. Leveille, Nucl. Phys. B137, 63 (1978).

[84] J. Grange et al. (Muon g-2 Collaboration), arXiv:1501 .06858 .

[85] R. H. Parker, C. Yu, W. Zhong, B. Estey, and H. Mller, Science 360, 191 (2018). 
[86] E. W. Kolb and M. S. Turner, Front. Phys. 69, 1 (1990).

[87] P. Gondolo and G. Gelmini, Nucl. Phys. B360, 145 (1991).

[88] N. Aghanim et al. (Planck Collaboration), Astron. Astrophys. 641, A6 (2020).

[89] P. A. R. Ade et al. (Planck Collaboration), Astron. Astrophys. 594, A13 (2016).

[90] A. Airapetian et al. (HERMES Collaboration), Phys. Rev. D 75, 012007 (2007).

[91] E. S. Ageev et al. (Compass Collaboration), Phys. Lett. B 647, 330 (2007).

[92] F. E. Maas and K. D. Paschke, Prog. Part. Nucl. Phys. 95, 209 (2017).

[93] J. Engel, Phys. Lett. B 264, 114 (1991).

[94] H.-Y. Cheng, Nucl. Phys. B, Proc. Suppl. 246-247, 109 (2014).

[95] A. L. Fitzpatrick, W. Haxton, E. Katz, N. Lubbers, and Y. $\mathrm{Xu}$, J. Cosmol. Astropart. Phys. 02 (2013) 004.

[96] N. Anand, A. L. Fitzpatrick, and W. C. Haxton, Phys. Procedia 61, 97 (2015).

[97] J. Walecka, in Semileptonic Weak Interactions in Nuclei (Academic Press, New York, 1975), Chap. V, Section IV, pp. 113-218.

[98] J. D. Lewin and P. F. Smith, Astropart. Phys. 6, 87 (1996).

[99] R. Catena and B. Schwabe, J. Cosmol. Astropart. Phys. 04 (2015) 042.
[100] E. Aprile et al. (XENON Collaboration), Phys. Rev. Lett. 122, 141301 (2019).

[101] X. Cui et al. (PandaX-II Collaboration), Phys. Rev. Lett. 119, 181302 (2017).

[102] R. Agnese et al. (CDMS Collaboration), Phys. Rev. Lett. 111, 251301 (2013).

[103] A. H. Abdelhameed et al. (CRESST Collaboration), Phys. Rev. D 100, 102002 (2019).

[104] J. L. Feng, Annu. Rev. Astron. Astrophys. 48, 495 (2010).

[105] E. Richard et al. (Super-Kamiokande Collaboration), Phys. Rev. D 94, 052001 (2016).

[106] P. Desiati (IceCube Collaboration), J. Phys. Conf. Ser. 120, 052038 (2008).

[107] T. Gaisser and M. Honda, Annu. Rev. Nucl. Part. Sci. 52, 153 (2002).

[108] T. J. Weiler, Phys. Rev. Lett. 49, 234 (1982).

[109] E. Roulet, Phys. Rev. D 47, 5247 (1993).

[110] D. A. Pierre, Optimization Theory with Applications, 1st ed. (Dover Publications, New York, 1986).

[111] S. G. Johnson, The nlopt nonlinear-optimization package, http://github.com/stevengj/nlopt.

[112] R. Agnese et al. (SuperCDMS Collaboration), Phys. Rev. D 95, 082002 (2017).

[113] F. Ruppin, J. Billard, E. Figueroa-Feliciano, and L. Strigari, Phys. Rev. D 90, 083510 (2014). 